\title{
EL ARTE Y LA EDUCACIÓN ARTÍSTICA EN CONTEXTOS DE SALUD
}

\section{Art and Art Education in health context}

\author{
Manuel H. Belver \\ Universidad Complutense de Madrid \\ mhbelver@art.ucm.es
}

Recibido: 30 de junio de 2011

Aprobado: 25 de julio de 2011

\section{Resumen:}

En este trabajo se analizan actividades artísticas desarrolladas en contextos de salud. Se hace una revisión de las investigaciones presentadas en el volumen, en línea con las tendencias más recientes en arte y salud.

Palabras clave: arte, salud, creatividad, niños, adolescentes, Alzheimer, demencias.

Belver, M. H. 2011: El Arte y la Educación artística en contextos de salud. Arte, Individuo y Sociedad. Vol. 23, Núm. Especial, 11-17.

\section{Abstract:}

In this work, the artistic activities carried out in health settings are analyzed. A review of the investigations presented in the volume is provided, along the most recent research tendencies in art and health. Key words: art, health, creativity, children, adolescents, Alzheimer, dementias. 
La consideración que se da a las actividades artísticas en el ámbito del cuidado y la atención a la salud es muy parecida a la que se les ha prestado tradicionalmente en la educación convencional: en general, estas actividades son consideradas agradables pero no necesarias. Sin embargo, las experiencias que presentamos en este volumen, y que están en la línea de las investigaciones más recientes, nos llevan a concluir que las artes, además de agradables, pueden ser más necesarias de lo que habitualmente suponemos. Hay una vía intermedia entre los planteamientos que encasillan el arte dentro de las actividades superfluas del ser humano, y por tanto prescindibles, y aquellos que lo consideran una plataforma a través de la cual arreglar muchos males de la humanidad. Tanto una como otra postura tienen sus defensores y detractores, pero ambas adolecen de extremas, pues ni el arte es prescindible, ni el artista tiene que ser la persona más indicada para mejorar el mundo (Kuspit, 2006).

Pero el arte y los artistas pueden contribuir, dentro de sus posibilidades, a hacer más llevaderas las condiciones de vida de muchas personas, proporcionarles medios para sentirse mejor consigo mismo, aislarse de ambientes poco agradables, adquirir autoconfianza, sentirse un poco más felices. El objetivo del arte no es curar, pero puede ayudar a humanizar los procesos de curación y los entornos en los que estos se desarrollan.

Eisner, de sobra conocido en el campo de la educación artística, señala varias funciones cognitivas que desempeñan las artes y que, según él, afectan a la conciencia (Eisner, 2004). Estas funciones estarían relacionadas con la sensibilidad, la imaginación y la representación. Para este autor,

las artes "refinan nuestros sentidos para que nuestra capacidad de experimentar el mundo sea más compleja y sutil; estimulan el uso de nuestra imaginación (...); nos ofrecen modelos para que podamos experimentar el mundo de nuevas maneras; $y$ nos proporcionan materiales y ocasiones para que podamos abordar problemas que dependen de formas de pensamiento relacionadas con las artes. También celebran los aspectos consumativos y no instrumentales de la experiencia humana y proporcionan medios para poder expresar significados inefables pero que se pueden sentir"(p. 38).

Los procesos relacionados con la sensibilidad, la imaginación y la representación, incluida la comunicación, que son los que Eisner señala, pueden jugar un papel importante en la salud de las personas, entendida esta, no solo como ausencia de enfermedad, sino como bienestar físico, psicológico y social, y en ello coincide con otro autor clásico, Vigotski, que a lo largo de su obra resaltó la importancia de la creatividad y la imaginación en los procesos cognitivos humanos (Vygotski, 2007).

Eisner se plantea como uno de los objetivos de su libro "El arte y la creación de la mente" rebatir la noción de que

"las artes, de algún modo, son operaciones realizadas con las manos que plantean pocas exigencias desde el punto de vista intelectual, que son más emotivas que reflexivas, que tienen poco que ver con la mente" (p.14), 
y propone una noción completamente diferente:

"la capacidad de crear una forma de experiencia que se pueda considerar estética requiere una mente que anime nuestra capacidad de imaginación y que estimule nuestra capacidad de vivir experiencias saturadas de emociones" ( $p .14)$.

Porque, en definitiva,

"las artes son medios para explorar nuestro paisaje interior. Cuando las artes nos conmueven de una manera genuina, descubrimos lo que somos capaces de experimentar. En este sentido, las artes nos ayudan a descubrir el contorno de nuestro ser emocional. Ofrecen recursos para experimentar el alcance y la variedad de nuestra receptividad y nuestra sensibilidad" (p. 28).

"las artes son medios para explorar nuestro paisaje interior. Cuando las artes nos conmueven de una manera genuina, descubrimos lo que somos capaces de experimentar. En este sentido, las artes nos ayudan a descubrir el contorno de nuestro ser emocional. Ofrecen recursos para experimentar el alcance y la variedad de nuestra receptividad y nuestra sensibilidad" (p. 28).

El panorama de investigación e intervención en arte y salud en España no alcanza el nivel de otros países ((Clift et al., 2009; Cox et al., 2010; Cuypers et al., 2011; Sonke, Rollins, Brandman, \& Graham-Pole, 2009; Wreford, 2010). Sin embargo, van apareciendo experiencias innovadoras en diferentes entornos, tanto de investigación como en contextos específicos de cuidado de la salud. Las experiencias que componen este volumen se caracterizan por integrar el arte, las actividades creativas y los productos artísticos como parte de los procesos de atención y cuidado a personas en situación de especial vulnerabilidad. Varios de los trabajos están dedicados a exponer experiencias desarrolladas en el contexto del proyecto Curarte $i+d$ (www.ucm.es/ info/curarte). Este proyecto, con casi una década de existencia, es pionero en España (Ullán, 2005; Ullán \& Belver, 2006). A partir de la creación de un grupo de investigación multidisciplinar de las Universidades de Salamanca y Complutense de Madrid, se han desarrollado un buen número de iniciativas en hospitales y centros de salud con niños y adolescentes y, recientemente, también con personas con enfermedad de Alzheimer y otras demencias, y de las cuales se incluye en el volumen una muestra representativa. Afortunadamente, otras iniciativas similares están produciéndose en nuestro país, y la intención de este número especial de AIS es hacerlas más visibles y difundirlas entre los colectivos interesados. Todas han sido realizadas en los últimos años, y muchas de ellas continúan realizándose en la actualidad. Esta es una de las características de este tipo de actividades: no son propuestas puntuales, sino actividades que puedan consolidarse y aportar una mejora real a los numerosos usuarios potenciales que pasan por estos centros.

En el caso de los niños y adolescentes hospitalizados, se ha observado que las actividades artísticas pueden contribuir a mejorar su experiencia de hospitalización, ofreciendo la posibilidad de ocupar el tiempo de su estancia de manera creativa y normalizadora. Ya en trabajos anteriores (Belver \& Ullán, 2010, 2011; Ullán \& Manzanera, 2009) se habían estudiado estos factores desde varias perspectivas. En el presente volumen se incorporan trabajos que consolidan este enfoque, e incorporan 
obras de artistas contemporáneos o las actividades de los propios artistas. Así, el trabajo de Moreno, Abad y Méndez, que utilizan obras de artistas como Chema Madoz, Victoria Encinas, Yayoi Kusama o Liliana Porter para su propuesta de talleres de arte contemporáneo con material reciclado con niños hospitalizados, o los de Gutiérrez, Megías y Nuere, con el juego de construcción de casas de muñecas como pretexto para recrear (construyendo) el propio hospital e interactuar con él de manera positiva, y el de Cuesta, Gómez y Megías sobre los talleres realizados en el MuPAI dentro de las actividades de verano, y su extrapolación y adaptación, a partir de esa experiencia, a otros similares en el Hospital Gregorio Marañón de Madrid, donde el Proyecto Curarte lleva trabajando desde hace años. Otra propuesta realizada a partir del arte contemporáneo es la de Megías et al., en la que son las propias artistas participantes las que realizan las intervenciones de los talleres con adolescentes del Servicio de Psiquiatría del Hospital Gregorio Marañón.

Un artista con una larga trayectoria en intervenciones de este tipo es Josep María Martín, con quien establecimos el primer contacto con motivo de la exposición en ARCO 2008 de su "prototipo de espacio para gestionar las emociones" en el hospital de Castellón, y del que habla extensamente, así como de la implicación del artista en este tipo de proyectos que pueden ayudar a los pacientes y sus familiares, en la entrevista realizada por M. G. Cano, incluida también en este volumen.

También la mediación de los artistas para ayudar a combatir la angustia o el dolor, así como el beneficio que puede suponer el proceso creativo para los propios artistas, son aspectos sobre los que reflexiona L. Manonelles en su trabajo, en el que aporta experiencias de artistas como Antonio Ortega o Carlos Pina.

Como iniciativa novedosa en este ámbito se presenta la del hospital de Denia, donde es el propio hospital el que promueve un proyecto en el que el arte tiene un papel destacado. El Proyecto CuidArt incorpora también la participación de artistas, con exposiciones, intervenciones de arte en vivo, talleres para pacientes y personal sanitario, etc., en un tipo de propuesta que incluye la colaboración con la Facultad de Bellas Artes de la Universidad Politécnica de Valencia a través de la cátedra DKV Arte y Salud, en la que se proporcionan becas de apoyo a jóvenes artistas en este campo, y garantiza una línea de investigación sólida en este ámbito.

Otro foco de actividad en los hospitales son las aulas hospitalarias, garantes del derecho de los pacientes pediátricos a la continuidad educativa, dentro de sus limitaciones, sin que la estancia en el hospital les suponga un retraso insalvable cuando se reincorporan. Pero además, estas aulas son verdaderos refugios para que los niños puedan evadirse, aunque sea por un rato, de la rutina del hospital (Ullán \& Belver, 2008). Por eso son importantes, además de sus actividades normales, otras que ayuden a los niños y adolescentes a mejorar su estancia. Este es el caso del trabajo de (Julia), representativo de lo que puede hacerse en y desde estas aulas.

También se están realizando proyectos multidisciplinares en hospitales a partir de la iniciativa de colectivos o asociaciones como la gallega sen marco, que presenta el trabajo Algo más de color, realizado en el área de pediatría del Complejo Hospitalario Universitario de Santiago de Compostela por Ante, González, López, Peleteiro, Rodríguez y Ruibal, con la colaboración de la artista Mónica Alonso. 
La variedad de propuestas posibles está representada, además, por trabajos que van desde la utilización del estampado no tóxico con niños y adolescentes discapacitados, realizado por Martínez y Albar, o las técnicas de creatividad a partir de la obra de artistas contemporáneos, de R. Herrero, a la utilización también de imágenes de arte contemporáneo como generadoras de distracción positiva en niños hospitalizados, de M. G. Cano, o el uso del cine con niños y adolescentes hospitalizados, en los talleres realizados por Antúnez y Méndez, en los que, a partir de los resultados de experiencias similares en el MuPAI (talleres de verano "Vacaciones de cine"), los pacientes realizaron una serie de cortometrajes que fomentaran su participación y mejorasen su autoestima.

La creación de objetos y su personalización a través del "tuneado" de los mismos por los adolescentes hospitalizados es otra de las propuestas presentadas, por Zapatero y Agudín, dentro del Proyecto Curarte, así como el de Perandones, que se enmarca en el mundo de los videojuegos y las posibilidades de los mismos para trabajar con estos pacientes.

Un ámbito de actuación que se ha desarrollado mucho en los últimos años ha sido el de los museos, que a sus iniciativas tradicionales han incorporado otras dirigidas a integrar de manera activa a colectivos que, por diversas causas, tienen menos acceso a estas instituciones, como es el de las personas con problemas de salud. En este contexto cabe destacar el trabajo realizado con pacientes del Hospital de Día de Adicciones de Álava entre este hospital y el ARTIUM de Vitoria, con la colaboración de la artista Susana Talayero, en torno a la exposición Miedo, con talleres y actividades fuera de las habituales en estos pacientes, y que fomentan la participación y el trabajo colaborativo en ellos, o el llevado a cabo en el Museo Thyssen Bornemisza de Madrid por su área de Investigación y Extensión educativa, a través de las líneas de educación y acción en las que se enmarcan los programas de este museo para públicos con necesidades especiales y Red de Públicos, donde tienen una especial relevancia las líneas relacionadas con el área de salud, como se presenta en el trabajo de Gamoneda.

En esta línea de iniciativas por parte de los museos se inscribe la de Es Baluard, el Museu d'Art Modern i Contemporani de Palma, presentado por Eva Cifre, que en ese propósito de acercar el arte contemporáneo a distintos colectivos, presenta los talleres realizados por la artista Isabel Castro con pacientes de la Unidad de Media Estancia "Lluerna", del Área Hospitalaria de Salud Mental de Gesma, y el taller "Fes el teu autoretrat" ("Haz tu autorretrato"), con pacientes de la Unidad Pediátrica de Semicríticos del Hospital Universitario Son Dureta, proyecto de Virginia Vallés, en el marco del proyecto "Red de intercambio entre programas de educación especial de centros de arte contemporáneo de la Eurorregión Pirineos-Mediterráneo", y del que se presentan también en este volumen las demás experiencias.

En el ámbito de la atención a las personas con demencia, se observa que la práctica artística tiene capacidad para desarrollar habilidades y estrategias que pueden contribuir a mejorar la adaptación de las personas a su propia condición clínica de demencia, e incluso el arte puede ayudar a que los cuidadores mejoren sus habilidades y las estrategias de cuidado. En este sentido se han empezado a desarrollar en los últimos años propuestas que inciden en las posibilidades de las actividades artísticas 
con estos colectivos. Prueba de ello son los trabajos de Barquín y Moreno, que desde el centro de Arte y Naturaleza (CDAN) de Huesca presentan un proyecto de arte contemporáneo en el hospital, a través de un taller de fotografía llevado a cabo por la artista Vicky Méndiz con enfermos mentales, y la de Ullán, codirectora del Proyecto Curarte $\mathrm{i}+\mathrm{d}$ y que presenta una nueva línea de este proyecto, el proyecto AR.S (arte y salud), centrado en las actividades realizadas con pacientes del Centro de Referencia Estatal de atención a personas con enfermedad de Alzheimer y otras demencias de Salamanca, y en las que han participado miembros del equipo Curarte.

Todas estas experiencias avalan la necesidad de la profesionalización de las personas que están dedicadas o pueden dedicarse de manera especializada a las actividades artísticas en los contextos de salud, como plantea el trabajo de Ávila y Acaso. La formación de profesionales en este contexto interdisciplinar del arte y la salud contribuiría a reforzar y mejorar la labor que ya se está haciendo, y dotaría a los centros de personal cualificado para realizar este tipo de tareas, dando estabilidad a los programas y mejorando cuestiones todavía pendientes en España, como la evaluación sistemática de este tipo de intervenciones, en línea con las tendencias a nivel internacional.

\section{Referencias}

Belver, M. H., \& Ullán, A. M. 2010: Symbolic environmental mediators in health settings: The role of art in the humanization of children's hospitals. Arte, Individuo y Sociedad, 22(2), 73-81.

Belver, M. H., \& Ullán, A. M. 2011: Art in a Spanish children's hospital. Arts \& Health, 3(1), 73-83. doi: 10.1080/17533015.2010.525516

Clift, S., M. Camic, P., Chapman, B., Clayton, G., Daykin, N., Eades, G., . . White, M. 2009: The state of arts and health in England. Arts \& Health, 1(1), 6-35. doi: 10.1080/17533010802528017

Cox, S. M., Lafrenière, D., Brett-MacLean, P., Collie, K., Cooley, N., Dunbrack, J., \& Frager, G. 2010: Tipping the iceberg? The state of arts and health in Canada. Arts \& Health, 2(2), 109-124. doi: 10.1080/17533015.2010.481291

Cuypers, K. F., Knudtsen, M. S., Sandgren, M., Krokstad, S., Wikström, B. M., \& Theorell, T. r. 2011: Cultural activities and public health: research in Norway and Sweden. An overview. Arts \& Health, 3(1), 6-26. doi: 10.1080/17533015.2010.481288

Eisner, E. W. 2004: El arte y la creación de la mente. Barcelona: Paidós.

Kuspit, D. 2006: El fin del arte. Madrid: Akal.

Sonke, J., Rollins, J., Brandman, R., \& Graham-Pole, J. 2009: The state of the arts in healthcare in the United States. Arts \& Health, 1(2), 107-135. doi: 10.1080/17533010903031580 
Ullán, A. M. 2005: Arte y creatividad infantil en los contextos hospitalarios. In M. H. Belver, M. Acaso \& I. Merodio (Eds.), Arte infantil y Cultura visual (pp. 113-129). Madrid: Eneida.

Ullán, A. M., \& Belver, M. H. 2006: Jugar para estar mejor: el juego de los niños en los hospitales. In M. H. Belver \& A. M. Ullán (Eds.), La creatividad a través del juego. Salamanca: Amarú ediciones.

Ullán, A. M., \& Belver, M. H. 2008: Cuando los pacientes son niños. Humanización y calidad en la hospitalización pediátrica. Madrid: Eneida.

Ullán, A. M., \& Manzanera, P. 2009: Las paredes cuentan: arte para humanizar un espacio de salud pediátrico. Arte, Individuo y Sociedad, 21, 123-142.

Vygotski, L. S. 2007: La imaginación y la creatividad en la infancia. In P. del Río \& A. Álvarez (Eds.), Escritos sobre arte y educación creativa de Lev S. Vygotski. Madrid: Fundación Infancia y aprendizaje.

Wreford, G. 2010: The state of arts and health in Australia. Arts \& Health, 2(1), 8-22. doi: 10.1080/17533010903421484 